\title{
THE RECIPROCITY FORMULA FOR MULTIDIMENSIONAL THETA FUNCTIONS
}

\author{
RICHARD BELLMAN AND R. SHERMAN LEHMAN
}

1. Introduction. The reciprocity formula for multidimensional theta functions is usually proved either by using the Poisson summation formula or by induction on the dimension starting with the one-dimensional case

$$
\sum_{k=-\infty}^{\infty} \exp \left(-\pi k^{2} t+2 \pi i k x\right)=t^{-1 / 2} \sum_{k=-\infty}^{\infty} \exp \left(-\pi(k+x)^{2} / t\right) .
$$

In this paper we shall give a proof based on the following simple idea: An identity can be proved by first finding a set of conditions which uniquely determines the function represented by the expression on one side of the identity and then verifying that these conditions are satisfied by the function represented by the expression on the other side. ${ }^{1}$

Using vector-matrix notation, we let

$$
n=\left(\begin{array}{c}
n_{1} \\
n_{2} \\
\vdots \\
n_{N}
\end{array}\right), \quad z=\left(\begin{array}{c}
z_{1} \\
z_{2} \\
\vdots \\
z_{N}
\end{array}\right), \quad T=\left(\begin{array}{ccc}
t_{11} & t_{12} \cdots t_{1 N} \\
t_{21} & t_{22} \cdots t_{2 N} \\
& \cdots \\
t_{N 1} & t_{N 2} \cdots t_{N N}
\end{array}\right)
$$

with the components of $n$ integers, and the components of $z$ and the elements of $T$ complex numbers. We let $e_{k}$ be the unit $N$-dimensional vector with $k$ th component equal to 1 and all others equal to 0 . A prime ' will be used to denote transposition, and $|T|$ will denote the determinant of the matrix $T$. A column vector will be treated as a rectangular matrix with one column, and a $1 \times 1$ matrix will be identified with its single element, so that $z^{\prime} T z=\sum_{i=1}^{N} \sum_{j=1}^{N} t_{i j} z_{i} z_{j}$. We shall also use the following convention, which, at least in its simplest form, is common in matrix theory: If we have an expression which denotes a number and contains the letters " $i$ " and " $j$ " as subscripts, then we obtain an expression for an $N \times N$ matrix having this number as its $(i, j)$ element by enclosing the original expression in parentheses. Thus, for example, $\left(t_{i j}\right)$ is the matrix $T$ and $\left(e_{i}^{\prime} e_{j}\right)$ is the identity matrix $I$ having 1's on the main diagonal and 0 's elsewhere.

Received by the editors October 8, 1960 .

${ }^{1}$ This idea has, of course, been used by many mathematicians. For further references, see [1]. 
The formula

$$
\begin{aligned}
& \sum_{n} \exp \left(-\pi n^{\prime} T n+2 \pi i n^{\prime} z\right) \\
& \quad=|T|^{-1 / 2} \sum_{n} \exp \left(-\pi(n+z)^{\prime} T^{-1}(n+z)\right),
\end{aligned}
$$

where the sums are taken over all vectors $n$ with integer components, is the reciprocity formula for multidimensional theta functions. It is not difficult to establish that both sums converge absolutely for every complex vector $z$ if $T$ is a symmetric matrix whose real part is positive definite. Also, by a uniform-convergence argument one can prove that each side represents an analytic function of the variables $z_{i}$ $(1 \leqq i \leqq n)$ and $t_{i j}(1 \leqq i \leqq j \leqq n)$ for all $z$ and for all symmetric $T$ with positive definite real parts. We shall take the left hand side as our definition of a theta function,

$$
\theta(z, T)=\sum_{n} \exp \left(-\pi n^{\prime} T n+2 \pi i n^{\prime} z\right) .
$$

2. Derivatives with respect to a matrix variable. It will be convenient to have the following notion of a derivative with respect to a symmetric matrix variable.

DeFINITION 1. If $f(T)$ is a complex-valued function of the variable $T$ which ranges over symmetric matrices, then we set

$$
\frac{d}{d T} f(T)=\left(\eta_{i j} \frac{\partial f(T)}{\partial t_{i j}}\right)
$$

where $\eta_{i j}=1$ if $i=j$ and $\eta_{i j}=1 / 2$ if $i \neq j$.

Observe that $d / d T$ is applied to scalar-valued functions, but that the result of the operation is an $N \times N$ matrix function. The operator $d / d T$ was introduced by Gårding [2] in a study of hyperbolic partial differential equations. It has also been used by Koecher [3] and Maass [4] in the study of Siegel's modular functions.

This formal derivative has many of the properties of an ordinary derivative. It is a linear operator; the product rule

$$
\frac{d}{d T}(f(T) g(T))=\frac{d f(T)}{d T} g(T)+f(T) \frac{d g(T)}{d T}
$$

is valid; and if $\phi$ is a function which maps complex numbers into complex numbers, then

$$
\frac{d}{d T}(\phi(f(T)))=\dot{\phi}(f(T)) \frac{d f(T)}{d T}
$$


where $\dot{\phi}$ is the ordinary derivative of $\phi$.

LEMMA 1. If $T$ is nonsingular, then

$$
\frac{d}{d T}|T|=|T| T^{-1}
$$

Proof. Let $X=\left(x_{i j}\right)$ be a matrix in which the $N^{2}$ elements are all independent variables, and let $X_{i j}$ be the cofactor of $x_{i j}$. Then, since

$$
|X|=\sum_{k=1}^{N} x_{i k} X_{i k}
$$

we have

$$
\frac{\partial|X|}{\partial x_{i j}}=X_{i j}
$$

Now let $x_{i j}=x_{j i}=t_{i j}(1 \leqq i \leqq j \leqq N)$. Then

$$
\begin{aligned}
\frac{\partial|X|}{\partial t_{i i}} & =X_{i i}, \\
\frac{1}{2} \frac{\partial|X|}{\partial t_{i j}} & =\frac{1}{2} \frac{\partial|X|}{\partial x_{i j}}+\frac{1}{2} \frac{\partial|X|}{\partial x_{j i}}=X_{j i} \quad(i \neq j) .
\end{aligned}
$$

The lemma then follows from the relation $T^{-1}=X^{-1}=\left(X_{j i} /|X|\right)$.

Another way to calculate derivatives of this sort is to go back to the limit definition of a derivative. Let $k$ and $l$ be fixed integers with $1 \leqq k \leqq N, 1 \leqq l \leqq N$. We define a matrix $U_{k l}$ whose $(i, j)$ element $u_{i j}$ is given by the formula

$$
u_{i j}=\left\{\begin{array}{cl}
1 & \text { if } i=j=k=l \\
1 / 2 & \text { if } i=k, j=l, k \neq l \\
1 / 2 & \text { if } i=l, j=k, k \neq l \\
0 & \text { otherwise. }
\end{array}\right.
$$

Thus, if $N=2$, we have

$$
U_{11}=\left(\begin{array}{ll}
1 & 0 \\
0 & 0
\end{array}\right), \quad U_{12}=U_{21}=\left(\begin{array}{cc}
0 & 1 / 2 \\
1 / 2 & 0
\end{array}\right), \quad U_{22}=\left(\begin{array}{ll}
0 & 0 \\
0 & 1
\end{array}\right) \text {. }
$$

The matrix $d f(T) / d T$ is given by the equation

$$
\frac{d}{d T} f(T)=\left(\lim _{\epsilon \rightarrow 0} \frac{f\left(T+\epsilon U_{i j}\right)-f(T)}{\epsilon}\right) .
$$


LEMMA 2. If $v$ is a fixed vector and $T$ is nonsingular, then

$$
\frac{d}{d T} v^{\prime} T^{-1} v=-\left(v^{\prime} T^{-1} U_{i j} T^{-1} v\right)
$$

Proof. If $\epsilon$ is sufficiently small in absolute value, then

$$
\begin{aligned}
\left(T+\epsilon U_{i j}\right)^{-1} & =T^{-1}\left(I+\epsilon U_{i j} T^{-1}\right)^{-1} \\
& =T^{-1}\left(I-\epsilon U_{i j} T^{-1}+\epsilon^{2}\left(U_{i j} T^{-1}\right)^{2}-\cdots\right) .
\end{aligned}
$$

Hence

$$
\lim _{\epsilon \rightarrow 0} \frac{v^{\prime}\left(T+\epsilon U_{i j}\right)^{-1} v-v^{\prime} T^{-1} v}{\epsilon}=-v^{\prime} T^{-1} U_{i j} T^{-1} v .
$$

The set of all symmetric $N \times N$ matrices can be regarded as an $N(N+1) / 2$-dimensional Euclidean space by taking the elements on and above the main diagonal as coordinates. Let $\Omega$ be an open connected set of symmetric matrices. The following lemma is just a translation of a theorem of the differential calculus for functions of more than one variable.

Lemma 3. If $d f(T) / d T=0$ for all $T$ in $\Omega$, then there is a constant $c$ such that $f(T)=c$ for all $T$ in $\Omega$.

To simplify some of the formulas we also make the following definition.

DEFINITION 2. If $f(z)$ is a complex-valued function of the vector variable $\mathrm{z}$, we let

$$
\frac{d^{2} f(z)}{d z^{2}}=\left(\frac{\partial^{2} f(z)}{\partial z_{i} \partial z_{j}}\right)
$$

3. A uniqueness theorem. The idea of characterizing a function not by a single expression but by a set of conditions was a favorite of Riemann's. In [5] Riemann listed conditions which determine $\theta(z, T)$ uniquely except for a factor independent of $z$. The following known theorem (see Encyklopädie der mathematischen Wissenschaften, vol. 2, part 2, pp. 637-638) provides conditions which uniquely determine $\theta(z, T)$ except for a constant factor.

THEOREM 1. Suppose that $f(z, T)$ is an entire function of $z$ satisfying the conditions

(i) $f\left(z+e_{k}, T\right)=f(z, T)$ $(k=1,2, \cdots, N)$,

(ii) $f\left(z+i T e_{k}, T\right)=\exp \left(-2 \pi i e_{k}^{\prime} z+\pi e_{k}^{\prime} T e_{k}\right) f(z, T)(k=1,2, \cdots, N)$. Then $f(z, T)=g(T) \theta(z, T)$ where $g(T)$ is independent of $z$. If in addition the differential equation 


$$
\left.\frac{d^{2} f(z, T)}{d z^{2}}\right|_{z=0}=4 \pi \frac{d f(0, T)}{d T}
$$

is satisfied for all symmetric matrices $T$ which are positive definite, then for all $\mathrm{z}$ and all positive definite symmetric $T$

$$
f(z, T)=c \theta(z, T)
$$

where $c$ is a constant independent of $z$ and $T$.

Proof. Let $\zeta_{k}=\exp \left(2 \pi i z_{k}\right) \quad(k=1,2, \cdots, N)$ and consider the function $\Phi\left(\zeta_{1}, \zeta_{2}, \cdots, \zeta_{N}\right)=f(z, T)$. The function $\Phi$ is by the hypothesis of the theorem a single-valued function of $\zeta_{1}, \zeta_{2}, \cdots, \zeta_{N}$ which is analytic for

$$
0<\left|\zeta_{k}\right|<\infty \quad(k=1,2, \cdots, N) .
$$

Consequently $\Phi$ has a Laurent expansion

$$
\Phi\left(\zeta_{1}, \zeta_{2}, \cdots, \zeta_{N}\right)=\sum_{n_{1}=-\infty}^{\infty} \cdots \sum_{n_{N}=-\infty}^{\infty} a_{n_{1} n_{2}} \cdots n_{N} \zeta_{1}^{n_{1}} \zeta_{2}^{n_{2}} \cdots \zeta_{N}^{n_{N}}
$$

and therefore $f$ has the Fourier expansion

$$
f(z, T)=\sum_{n} a_{n} \exp \left(2 \pi i n^{\prime} z\right)
$$

which converges absolutely and uniformly if $z$ is restricted to a bounded set.

Regarding $T$ as fixed, we now use the condition (ii) to compute the Fourier coefficients $a_{n}$. We must have

$$
\begin{aligned}
\sum_{n} a_{n} \exp \left(2 \pi i n^{\prime} z-2 \pi n^{\prime}\right. & \left.T e_{k}\right) \\
& =\exp \left(-2 \pi i e_{k}^{\prime} z+\pi e_{k}^{\prime} T e_{k}\right) \sum_{n} a_{n} \exp \left(2 \pi i n^{\prime} z\right) \\
& =\exp \left(\pi e_{k}^{\prime} T e_{k}\right) \sum_{n} a_{n+e_{k}} \exp \left(2 \pi i n^{\prime} z\right) .
\end{aligned}
$$

Hence we must have

$$
\begin{aligned}
a_{n+e_{k}} & =a_{n} \exp \left(-2 \pi n^{\prime} T e_{k}-\pi e_{k}^{\prime} T e_{k}\right) \\
& =a_{n} \exp \left(-\pi\left(n+e_{k}\right)^{\prime} T\left(n+e_{k}\right)+\pi n^{\prime} T n\right) \quad(k=1,2, \cdots, N) .
\end{aligned}
$$

The Fourier series is thus uniquely determined by $a_{0}$, i.e.

$$
f(z, T)=a_{0} \sum_{n} \exp \left(-\pi n^{\prime} T n+2 \pi i n^{\prime} z\right),
$$

where $a_{0}$ is independent of $z$. Taking $g(T)=a_{0}$, we obtain the first part of the theorem.

Now we regard $T$ as a variable and assume that the differential 
equation (3.1) is satisfied. Observing that

$$
\begin{aligned}
\frac{d}{d T} n^{\prime} T n & =\left(\lim _{\epsilon \rightarrow 0} \frac{n^{\prime}\left(T+\epsilon U_{i j}\right) n-n^{\prime} T n}{\epsilon}\right) \\
& =\left(n^{\prime} U_{i j} n\right)=\left(n_{i} n_{j}\right),
\end{aligned}
$$

we obtain

$$
\begin{aligned}
\frac{d f(0, T)}{d T} & =\frac{d}{d T}\left(g(T) \sum_{n} \exp \left(-\pi n^{\prime} T n\right)\right) \\
& =\sum_{n}\left\{\frac{d g(T)}{d T}-\pi\left(n_{i} n_{j}\right) g(T)\right\} \exp \left(-\pi n^{\prime} T n\right) .
\end{aligned}
$$

In this calculation, as in several others that follow, term by term differentiation is justified by appeal to the uniform convergence of the resulting series.

Also, since

$$
\frac{\partial^{2}}{\partial z_{i} \partial z_{j}} \exp \left(2 \pi i n^{\prime} z\right)=-4 \pi^{2} n_{i} n_{j} \exp \left(2 \pi i n^{\prime} z\right)
$$

we obtain

$$
\left.\frac{d f(z, T)}{d z^{2}}\right|_{z=0}=-4 \pi^{2} g(T) \sum_{n}\left(n_{i} n_{j}\right) \exp \left(-\pi n^{\prime} T n\right) .
$$

Combining (3.1)-(3.3) we find

$$
\left[\frac{d g(T)}{d T}\right] \sum_{n} \exp \left(-\pi n^{\prime} T n\right)=0 .
$$

The sum cannot vanish because it contains only positive terms. Hence $d g(T) / d T=0$.

We can now use Lemma 3 to prove that $g(T)$ is equal to a constant if we show that the set $\beta$ of positive definite symmetric matrices is an open connected subset of the space $\delta$ of symmetric matrices. One can prove that $P$ is open by using the determinantal criteria for positive definiteness. To see that $P$ is connected it is sufficient to show that it is convex. Let $S$ and $T$ be two matrices in $P, \lambda$ a real number between 0 and 1 , and $v$ a real vector. Then

$$
v^{\prime}(\lambda S+(1-\lambda) T) v=\lambda v^{\prime} S v+(1-\lambda) v^{\prime} T v>0 .
$$

The proof of the theorem is now complete.

4. Proof of the reciprocity formula. We now apply Theorem 1 to prove the reciprocity formula (1.2) for positive definite symmetric $T$. 
The formula can be extended to all symmetric $T$ with positive definite real parts by analytic continuation.

First we verify that the function

$$
\phi(z, T)=\sum_{n} \exp \left(-\pi(n+z)^{\prime} T^{-1}(n+z)\right)
$$

satisfies the periodicity conditions (i) and (ii). We have

$$
\begin{aligned}
\phi\left(z+e_{k}, T\right) & =\sum_{n} \exp \left(-\pi\left(n+z+e_{k}\right)^{\prime} T^{-1}\left(n+z+e_{k}\right)\right) \\
& =\sum_{m} \exp \left(-\pi(m+z)^{\prime} T^{-1}(m+z)\right)=\phi(z, T)
\end{aligned}
$$

where $m=n+e_{k}$ also runs over all vectors with integer components. Also

$$
\begin{aligned}
& \phi\left(z+i T e_{k}, T\right) \\
& =\sum_{n} \exp \left(-\pi\left(n+z+i T e_{k}\right)^{\prime} T^{-1}\left(n+z+i T e_{k}\right)\right) \\
& =\sum_{n} \exp \left(-\pi(n+z)^{\prime} T^{-1}(n+z)-2 \pi i e_{k}^{\prime} T T^{-1}(n+z)+\pi e_{k}^{\prime} T T^{-1} T e_{k}\right) \\
& =\exp \left(-2 \pi i e_{k}^{\prime} z+\pi e_{k}^{\prime} T e_{k}\right) \phi(z, T)
\end{aligned}
$$

because $e_{z}^{\prime} n$ is an integer. Thus $\phi(z, T)$ coincides with $\theta(z, T)$ except for a factor independent of $z$.

Next we determine $\beta(T)$ so that the function $\beta(T) \phi(z, T)$ satisfies the differential equation (3.1). It will then follow from Theorem 1 that there is a constant $c$ such that $\beta(T) \phi(z, T)=c \theta(z, T)$. We then will determine $c$ by a special choice of $T$.

We begin by computing derivatives of the terms in the series representation (4.1) for $\phi(z, T)$. Letting $u=(n+z)^{\prime} T^{-1}(n+z)$ and $\left(s_{i j}\right)=T^{-1}$, we have

$$
\begin{aligned}
\frac{\partial u_{.}}{\partial z_{j}} & =\frac{\partial}{\partial z_{j}} \sum_{k=1}^{N} \sum_{l=1}^{N} s_{k l}\left(n_{k}+z_{k}\right)\left(n_{l}+z_{l}\right)=2 \sum_{k=1}^{N} s_{k j}\left(n_{k}+z_{k}\right), \\
\frac{\partial^{2} u}{\partial z_{i} \partial z_{j}} & =2 s_{i j} .
\end{aligned}
$$

Hence using the matrices $U_{i j}$ introduced before Lemma 2, we obtain

$$
\begin{aligned}
\frac{d^{2}}{d z^{2}} & \exp (-\pi u) \\
& =\left(\frac{\partial^{2}}{\partial z_{i} \partial z_{j}} \exp (-\pi u)\right)=\left(-\pi \frac{\partial^{2} u}{\partial z_{i} \partial z_{j}}+\pi^{2} \frac{\partial u}{\partial z_{i}} \frac{\partial u}{\partial z_{j}}\right) \exp (-\pi u) \\
& =\left\{-2 \pi T^{-1}+\left(4 \pi^{2}(n+z)^{\prime} T^{-1} U_{i j} T^{-1}(n+z)\right)\right\} \exp (-\pi u) .
\end{aligned}
$$


Applying Lemma 2 we obtain

$$
\begin{aligned}
\frac{d}{d T}[\beta(T) \exp (- & \left.\left.\pi n^{\prime} T^{-1} n\right)\right] \\
& =\left\{\frac{d \beta(T)}{d T}+\beta(T)\left(\pi n^{\prime} T^{-1} U_{i j} T^{-1} n\right)\right\} \exp \left(-\pi n^{\prime} T^{-1} n\right) .
\end{aligned}
$$

Consequently, the differential equation (3.1) will be satisfied by $\beta(T) \phi(z, T)$ if $\beta(T)$ is a solution of

$$
\frac{d \beta(T)}{d T}=-\frac{1}{2} \beta(T) T^{-1}
$$

By Lemma 3 and the chain rule for differentiation given above, we see that $\beta(T)=|T|^{-1 / 2}$ yields a solution. Therefore

$$
\theta(z, T)=c|T|^{-1 / 2} \phi(z, T) \text {. }
$$

Next we set $T=I$ and observe that $\theta(0, I)=\phi(0, I) \neq 0$. Hence $c=1$, and the proof of the reciprocity formula is complete.

\section{REFERENCES}

1. R. Bellman, $A$ brief introduction to theta functions, New York, Holt, Rinehart and Co., 1961.

2. L.Gårding, The solution of Cauchy's problem for two totally hyperbolic linear differential equations by means of Riesz integrals, Ann. of Math. vol. 48 (1947) pp. 785-826.

3. M. Koecher, Ueber Thetareihen indefiniter quadratischer Formen, Math. Nachr. vol. 9 (1953) pp. 51-85.

4. H. Maass, Die Differentialgleichungen in der Theorie der Siegelschen Modulfunktionen, Math. Ann. vol. 126 (1953) pp. 44-68.

5. B. Riemann, Theorie der Abel'schen Funktionen in Collected works, pp. 88-144, New York, Dover Publications, Inc., 1953.

RAND CORPORATION AND

University of Califoria, Berkeley 